(C2018 IEEE. Personal use of this material is permitted. Permission from IEEE must be obtained for all other uses, in any current or future media, including reprinting/republishing this material for advertising or promotional purposes, creating new collective works, for resale or redistribution to servers or lists, or reuse of any copyrighted component of this work in other works 


\section{Robust Multipath Detection by Intra- and Inter-Domain Fusion with Real-Time Capability}

\author{
Artem Gostishchev \\ RWTH Aachen University \\ 52062 Aachen, Germany \\ Email: artem.gostishchev@rwth-aachen.de
}

\author{
Friederike Fohlmeister \\ German Aerospace Center (DLR) \\ 82234 Wessling, Germany \\ Email: friederike.fohlmeister@dlr.de
}

\author{
Andriy Konovaltsev \\ German Aerospace Center (DLR) \\ 82234 Wessling, Germany \\ Email: andriy.konovaltsev@dlr.de
}

\begin{abstract}
-
This paper presents a domain fusion method for robust multipath detection in global navigation satellite systems (GNSS) receivers with antenna arrays. By combining the multipath detection results from different multipath detectors in the time and space domain, the method is able to compensate weaknesses of single-domain multipath detection methods in adverse conditions. In the time-domain, different features of the crosscorrelation function (CCF) are analyzed and intra-domain fusion is achieved by a classification approach. In the space-domain, several signal-number criteria are evaluated and intra-domain fusion is conducted using an evidence approach. The interdomain fusion between time-domain and space-domain features is conducted by joining the detection results from the different domains to either maximize the detection rate or minimize the false alarm rate. Experimental results indicate that the proposed approach outperforms conventional single-domain single-feature detection methods in simulated conditions.
\end{abstract}

\section{INTRODUCTION}

Global Navigation Satellite Systems (GNSS) have experienced an increasing interest during the last years. Apart from positioning and navigation on land, at sea and in the air, they are also used for timing purposes, for example in telecommunication and power supply networks. The modernization of existing and construction of newly designed systems, accompanied by corresponding space- and ground-based augmentation infrastructure creates the basis for high-precision applications [1].

The advance of GNSS applications into Safety-of-Life scenarios evokes their conceptual weaknesses, where the most harmful ones arise from ionospheric scintillation, interference and multipath propagation [1]. Many proposals how to detect, characterize and mitigate multipath have been made in the past. The approaches utilize different properties of the received signal. Some evaluate the signal shape in the time-domain [2], [3], others compare signal characteristics on different frequencies [4], [5]. Array-based methods perform spatial filtering of direct and indirect signals [6], [7] or exploit the polarization change in signal reflections [8], [9].

Any deviations from nominal signal conditions lead to a distortion of the cross-correlation function (CCF) between the received signal and its local replica. This feature is analyzed in Signal Quality Monitoring [10], but has also been proposed by Irsigler and Hein [11] and in works by Bhuiyan et al. [12], [13] for multipath analysis. Timedomain multipath mitigation is possible by non-parametric methods as for example the Narrow Correlator [14] or the High Resolution Correlator [15] techniques, which prevent multipath influence by using narrow-spaced correlators, or by combining outputs from multiple correlators. Parametric methods, for example NovAtel's MEDLL [16], often use maximum-likelihood approaches for asymptotic correctness, but suffer from increased computational complexity and their implementation in traditional receivers is not straight forward.

All time domain methods have in common that their performance is significantly reduced in the case of of high temporal correlation between the line-of-sight (LOS) and multipath signal. Such situations can therefore be not resolved in the time-domain, which motivates research towards arraybased receivers that may utilize the spatial diversity.

Array-based receivers use multiple antenna elements instead of one antenna and allow for signal processing in the spatial domain. This includes model order, i.e. number of signals estimation [17] and direction-of-arrival estimation [18], [19], [20]. However, all of these methods assume spatially uncorrelated signals. If this is not the case, i.e. the signals come from close directions, advances techniques, for example Forward-Backward-Averaging or Spatial Smoothing [21], allow to reduce spatial correlation, but also introduce constraints on signal phase relations and array geometries.

Although some of the mentioned approaches have already been implemented in receivers, the majority shows a low robustness in the cases of highly correlated LOS and multipath signals. Therefore, we propose a combined approach. In the time-domain, we use multiple CCF shape features based on [13], [22] and [11]. The intra-domain fusion is conducted by a combination with a total classification decision. This approach is known from information retrieval and machine learning [23]. In the space-domain, the intra-domain fusion 
of multiple signal-number estimators is conducted with the evidence based approach by Dempster and Shafer [24] to come up with a stabilized signal-number estimate. For the inter-domain fusion between the time-domain and the space-domain, we propose to use logical operators, which either maximize the detection or minimize the false alarm rate.

The proposed multipath detection approach is evaluated with software and hardware simulated GPS L1 C/A signals. Results show that the detection rate is increased for the intradomain fusion of different time domain methods and the false alarm rate is decreased for the intra-domain fusion of different space domain methods. In the case of temporally or spatially highly correlated LOS and multipath signal the multipath detection performance benefits from the inter-domain fusion.

\section{A. Notation}

In this paper, we define scalars, column vectors, and matrices with lowercase letters, lowercase bold letters, and uppercase bold letters, respectively. The $m$-th entry of a vector $\boldsymbol{a}$ is denoted $a_{m}$. The transpose and Hermitian (complex conjugation and transposition) of a matrix $\boldsymbol{A}$ are denoted $\boldsymbol{A}^{\mathrm{T}}$ and $\boldsymbol{A}^{\mathrm{H}}$, while $\boldsymbol{I}_{M}$ is the identity matrix of dimension $M \times M$.

\section{Signal MODEL}

Consider the complex baseband signal received by an antenna array with $M \in \mathbb{N}$ elements

$$
\boldsymbol{x}(t)=\boldsymbol{x}_{\mathbf{0}}(t)+\sum_{l=1}^{L} \boldsymbol{x}_{\boldsymbol{l}}(t)+\boldsymbol{\eta}(t)
$$

where $\boldsymbol{x}_{0}(t) \in \mathbb{C}^{M \times 1}$ is the LOS signal, and $\boldsymbol{x}_{\boldsymbol{l}}(t) \in$ $\mathbb{C}^{M \times 1}, l=1, \ldots L$ denote the $L \in \mathbb{N}$ multipath signals with

$$
\boldsymbol{x}_{\boldsymbol{l}}(t)=\gamma_{l} \boldsymbol{a}\left(\theta_{l}, \phi_{l}\right) c\left(t-\tau_{l}\right) e^{j 2 \pi f_{\mathrm{D}_{l}} t} \quad \forall l \in[0, L] .
$$

$\gamma_{l} \in \mathbb{C}$ denotes the complex signal amplitude and $\boldsymbol{a}\left(\theta_{l}, \phi_{l}\right) \in$ $\mathbb{C}^{M \times 1}$ denotes the array steering vector with elevation angle $\theta_{l} \in \mathbb{R}$ and azimuth angle $\phi_{l} \in \mathbb{R} . c\left(t-\tau_{l}\right) \in[-1,1]$ denotes the periodically repeated pseudo random (PR) sequence $c(t)$ with length $N_{\mathrm{c}}$, chip duration $T_{\mathrm{c}}$ and time delay $\tau_{l} \in \mathbb{R} . f_{\mathrm{D}_{l}} \in$ $\mathbb{R}$ accounts for the Doppler frequency, while $\boldsymbol{\eta}(t) \in \mathbb{C}^{M \times 1}$ is zero mean white Gaussian noise with covariance matrix $\boldsymbol{R}=$ $\sigma^{2} \boldsymbol{I}_{M}$. The exact value of $L$ is unknown, but to have sufficient degrees of freedom the total number of signals $D \in \mathbb{N}$ holds $D=1+L<M$. Sampling the signal at $f_{\mathrm{s}}=2 B=1 / T_{\mathrm{s}}$ and using the short notation $x\left(n T_{\mathrm{s}}\right)=x[n]$ yields

$$
\boldsymbol{x}[n]=\boldsymbol{x}_{\mathbf{0}}[n]+\sum_{l=1}^{L} \boldsymbol{x}_{\boldsymbol{l}}[n]+\boldsymbol{\eta}[n]
$$

with

$$
\boldsymbol{x}_{\boldsymbol{l}}[n]=\gamma_{l} \boldsymbol{a}\left(\theta_{l}, \phi_{l}\right) c\left(n T_{\mathrm{s}}-\tau_{l}\right) e^{j 2 \pi f_{\mathrm{D}_{l}} n T_{\mathrm{s}}} .
$$

\section{A. Post-Correlation Signal Model}

Correlating $N \in \mathbb{N}$ samples of the received signal $\boldsymbol{x}[n]$ with $N$ samples of a local code signal $\frac{1}{N} c\left(n T_{\mathrm{s}}-\hat{\tau}_{q}\right)$, where $\hat{\tau}_{q} \in \mathbb{R}$ is the delay of the replica signal, the output signal of the $q$-th correlator in the $k$-th epoch of length $T=N T_{\mathrm{s}}$ reads

$$
\boldsymbol{y}_{q}[k]=\boldsymbol{y}_{\mathbf{0}, \boldsymbol{q}}[n]+\sum_{l=1}^{L} \boldsymbol{y}_{\boldsymbol{l}, \boldsymbol{q}}[n]+\overline{\boldsymbol{\eta}}_{q}[n],
$$

with

$$
\boldsymbol{y}_{l, q}[n]=\gamma_{l} \boldsymbol{a}\left(\theta_{l}, \phi_{l}\right) \operatorname{sinc}\left(\frac{f_{\mathrm{D}_{l}} T}{2}\right) r\left(\tau_{l}-\hat{\tau}_{q}\right),
$$

$\overline{\boldsymbol{\eta}}_{q}$ being zero mean white Gaussian noise with covariance matrix $\overline{\boldsymbol{R}}=\frac{1}{N} \sigma^{2} \boldsymbol{I}_{M}$ and $r\left(\Delta_{\tau}\right) \in \mathbb{R}$ is the auto-correlation function of the PR sequence $c(t)$

$$
r\left(\Delta_{\tau}\right)=\frac{1}{N} c(t) * c\left(t-\Delta_{\tau}\right) .
$$

In (6) it is assumed that the integration time $T$ is an integer multiple of the code length $T_{\text {code }}$ of the PR sequence. Collecting the outputs of $Q \in \mathbb{N}$, where $Q$ is odd, different correlators with delays

$$
\hat{\tau}_{Q}=\left[\hat{\tau}_{1}, \ldots, \hat{\tau}_{P}, \ldots, \hat{\tau}_{Q}\right]^{\mathrm{T}}
$$

in one matrix we have

$$
\boldsymbol{Y}[k]=\left[\boldsymbol{y}_{1}[k], \ldots, \boldsymbol{y}_{Q}[k]\right] \in \mathbb{C}^{M \times Q} .
$$

The delay at the center, $P=\left\lfloor\frac{Q}{2}\right\rfloor+1$ takes the estimated value of the $\operatorname{LOS}$ delay $\tau_{0}$, i.e. the prompt correlator value. The $\left\lfloor\frac{Q}{2}\right\rfloor$ correlator delays of the early side are spaced within a maximum of one chip length $T_{\mathrm{c}}$ left of the prompt correlator, i.e. $\hat{\tau}_{q}<\hat{\tau}_{P} \forall q<P$. The $\left\lfloor\frac{Q}{2}\right\rfloor$ correlator delays of the late side are spaced within a maximum one chip length $T_{\mathrm{c}}$ right of the prompt correlator, i.e. $\hat{\tau}_{q}>\hat{\tau}_{P} \forall q>P$.

In a common GNSS receiver $\boldsymbol{Y}[k]$ is evaluated for $Q=3$ values of $\hat{\tau}_{q}$, i.e. the early, prompt and late correlator. In this case the prompt correlator value takes the estimated value of the LOS delay $\tau_{0}$, while the early and late correlator are equally spaced around the prompt correlator. To suppress multipath signals the spacing can be chosen small. In the work at hand, multipath should be detected. Therefore, a wide spacing is desirable. Moreover, as the CCF between received signal and local replica is analyzed, a higher number of samples is achieved by using more than three correlators. However, note that in contrast to the MEDLL [16] no further changes in the traditional receiver structure are necessary.

\section{B. Spatial Covariance Estimation}

To perform spatial multipath analysis, the spatial covariance matrix of $\boldsymbol{Y}[k], \boldsymbol{R}_{Y Y}[k] \in \mathbb{C}^{M \times M}$, must be estimated. This is done with the method proposed in [9], i.e.

$$
\boldsymbol{R}_{Y Y}[k]=\mathbb{E}\left\{\boldsymbol{Y}[k]\left(\boldsymbol{\Sigma} \boldsymbol{V}^{\mathrm{H}}\right)^{-1}\left(\boldsymbol{Y}[k]\left(\boldsymbol{\Sigma} \boldsymbol{V}^{\mathrm{H}}\right)^{-1}\right)^{\mathrm{H}}\right\},
$$


where the $\mathbb{E}\{\}$ operator denotes the expectation, and the matrices $\boldsymbol{\Sigma} \in \mathbb{R}^{Q \times Q}, \boldsymbol{V} \in \mathbb{R}^{Q \times Q}$ are obtained from the reduced singular value decomposition (SVD) of the matrix

$$
\boldsymbol{C}=\boldsymbol{U} \boldsymbol{\Sigma} \boldsymbol{V}^{\mathrm{H}}=\left[\begin{array}{ccc}
c\left(T_{\mathrm{s}}-\hat{\tau}_{1}\right) & \ldots & c\left(T_{\mathrm{s}}-\hat{\tau}_{Q}\right) \\
\vdots & \ddots & \vdots \\
c\left(N T_{\mathrm{s}}-\hat{\tau}_{1}\right) & \ldots & c\left(N T_{\mathrm{s}}-\hat{\tau}_{Q}\right)
\end{array}\right]
$$

The multiplication with $\left(\boldsymbol{\Sigma} \boldsymbol{V}^{\mathrm{H}}\right)^{-1}$ is a pre-whitening operation on $\boldsymbol{Y}[k]$ and decorrelates the noise over the columns of $\boldsymbol{Y}[k]$. Assuming an averaging time of $K_{p}$ observation periods and constant signal conditions, $\boldsymbol{R}_{Y Y}[k]$ can be estimated by

$$
\boldsymbol{R}_{Y Y}=\frac{1}{K_{p}} \sum_{k=1}^{K_{p}} \boldsymbol{Y}[k]\left(\boldsymbol{\Sigma} \boldsymbol{V}^{\mathrm{H}}\right)^{-1}\left(\boldsymbol{Y}[k]\left(\boldsymbol{\Sigma} \boldsymbol{V}^{\mathrm{H}}\right)^{-1}\right)^{\mathrm{H}} .
$$

\section{Methodology Description}

Having the received signal $\boldsymbol{Y}[k]$ in (9) the goal of multipath detection is to assign the received data to either of two classes of reception situations. One class is affected by multipath, while the other class represents multipath-free, i.e., LOS conditions. There are different multipath detection methods in the time and space domain. All methods analyze a certain feature, for example the shape of the received CCF or the estimated model order. Based on that a decision regarding the presence of multipath is generated, i.e., one of the trinary hypotheses

1 indicates multipath presence

$-1 \quad$ indicates no multipath, i.e., only LOS

0 indicates error or undecided,

is accepted. Using this framework allows the use of signs and logical operators to combine different hypotheses from different signal features to one robust decision.

\section{A. Time-domain Multipath Detection}

In the time-domain, a binary classification approach is applied. To assign the received data to either the multipath free, or the multipath situation, qualitative and quantitative features in the estimated CCF waveform are evaluated. For example in the case of the GPS LI C/A signal the ideal CCF is triangular in the interval $\left[-T_{C},+T_{C}\right]$ with unitary absolute slope, strictly monotonically increasing on the early side and strictly monotonically decreasing on the late side and no negative values. Additionally, the prompt correlator output should be the absolute maximum. The differentiated $\mathrm{CCF}$ is expected to have a zero transition and to be flat on both sides. As estimates for the CCF the inphase, i.e. real values of $\boldsymbol{Y}[k]$ are used. Further it is assumed that the data is wiped off. All time-domain features are also applicable for single antenna receivers. In the case of a multi-antenna array receivers they are applied row-wise on $\boldsymbol{Y}[k]$.
1) Qualitative Features: Qualitative features check the presence of strong CCF deviations and are collected in the qualitative feature vector $\boldsymbol{v}_{\mathrm{ql}} \in \mathbb{R}^{N_{\mathrm{ql}} \times 1}$, where $N_{\mathrm{ql}}$ is the number of considered qualitative features. Examples for qualitative features are negative inphase correlator values, additional extremes and invalid slopes. If such extremes are present, the corresponding entry of $\boldsymbol{v}_{\mathrm{q} l}$ is set to 1, otherwise the entry is set to 0 . Therefore, non-occurance of such extreme values is not regarded as no multipath, but no decision is made. The $N_{\mathrm{ql}}=7$ qualitative features used in this paper are summarized in Table III in the appendix.

2) Quantitative Features: Quantitative features describe either the relative asymmetry of the CCF shape on both sides, or measure the deviation from an ideal reference function. The feature extraction produces a quantitative feature vector $\boldsymbol{v}_{\mathrm{qn}} \in \mathbb{R}^{N_{\mathrm{qn}} \times 1}$, with $N_{\mathrm{qn}}$ denoting the number of quantitative features. The $N_{\mathrm{qn}}=9$ quantitive features used in this work are summarized in Table IV in the appendix. Features $v_{\mathrm{qn}_{1}}-v_{\mathrm{qn}_{5}}$, compare the absolute CCF slopes between different correlators on both sides. These features are derived from the proposals in [13] and [22], which are special cases of the general considerations in [10] and [11]. Features $v_{\mathrm{qn}_{6}}-v_{\mathrm{qn}_{9}}$ compare the slope and area differences between the estimated CCF and the ideal expectation. To be comparable, the quantitative features are normalized to their distribution parameters, i.e., to a normalized feature vector $\boldsymbol{v}_{\mathrm{nm}} \in \mathbb{R}^{N_{\mathrm{qn}} \times 1}$ with entries

$$
v_{\mathrm{nm}_{i}}=\sqrt{\frac{\left(v_{\mathrm{qn}_{i}}-v_{\mu_{i}}\right) \cdot\left(v_{\mathrm{qn}_{i}}-v_{\mu_{i}}\right)}{v_{\sigma_{i}}}},
$$

where it is assumed that the features are normally distributed. The vectors $\boldsymbol{v}_{\mu} \in \mathbb{R}^{N_{\mathrm{qn}} \times 1}$ and $\boldsymbol{v}_{\sigma} \in \mathbb{R}^{N_{\mathrm{qn}} \times 1}$ contain the distribution means and variances, respectively. They have to be determined empirically by receiver calibration. In this paper, we assume them to be given.

3) Hypothesis Test for Quantitative Features: To come up with a binary decision for quantitative features, the hypothesis test from [25] is applied where the normalized features $v_{\mathrm{nm}_{i}}$ are compared to thresholds $t_{\mathrm{h}_{i}} \in \mathbb{R}^{>0}$

$$
v_{\delta_{i}}=v_{\mathrm{nm}_{i}}-t_{\mathrm{h}_{i}}
$$

to find a decision. In the case $v_{\delta_{i}} \leq 0$ multipath free conditions are assumed, while in the case $v_{\delta_{i}}>0$ multipath reception is assumed. Since the features are assumed as normally distributed, the thresholds $t_{\mathrm{h}_{i}}$ can be selected directly from the properties of the normal distribution, based on the tolerable false alarm rate.

Having evaluated the qualitative and quantitative features in the time domain, a joint decision, including all features can be calculated either on feature- or decision level [26].

4) Intra-Domain Fusion of Quantitative Features on Feature Level: For the intra-domain fusion of the quantitative 
features we use that the norm of a vector depends on the absolute value of the vector elements. Since the normalized feature values $v_{\mathrm{nm}_{i}}$ are close to zero in the multipath-free case and higher in multipath affected conditions, a threshold $t_{\mathrm{q}}$ on the norm of the normalized feature vector $\boldsymbol{v}_{\mathrm{nm}}$ is used to generate the quantitative decision $d_{\mathrm{q}}$ by

$$
d_{\mathrm{q}}=\operatorname{sgn}\left(\sqrt{\sum_{i} v_{\mathrm{nm}_{i}}^{2}}-t_{\mathrm{q}}\right) \text {. }
$$

Since the vector norm is not normally distributed, the threshold $t_{\mathrm{q}}$ has to be either determined from analytic derivations or selected by calibration.

5) Intra-Domain Fusion of Quantitative Features on Feature Level for Multiple Antennas: Assuming uncorrelated noise at different antenna elements, a stabilized decision can be generated by consequently averaging over the quantitative features of all antenna elements. Given the quantitative feature vector $\boldsymbol{v}_{\mathrm{nm}, m}$ for $m=1, \ldots, M$ antennas, the fusion of quantitative features discussed in section III-A4 can be combined for all $M$ antennas of the array. Let $\boldsymbol{v}_{\mathrm{nm}, m}$ denote the feature vector of the $m$-th antenna element, $t_{\mathrm{q}_{m}}$ denote the corresponding threshold then we can calculate

$$
\delta_{m}=\sqrt{\sum_{i} v_{\mathrm{nm}, m_{i}}^{2}}-t_{\mathrm{q}_{m}}
$$

The sign of $\delta_{m}$ corresponds to the quantitative decision $d_{\mathrm{q}}$ in (15). The averaged decision between consequent elements is

$$
d_{\mathrm{q}_{m}}=\operatorname{sgn}\left(\frac{\delta_{m}+\delta_{m+1}}{2}\right) .
$$

Cyclic application yields a decision vector $\boldsymbol{v}_{a} \in \mathbb{R}^{M \times 1}$ with elements

$v_{\mathrm{a}_{i+1}}=\operatorname{sgn}\left(\frac{\delta_{i \bmod M}+\delta_{(i+1) \bmod M}}{2}\right) \quad i \in[0, M-1]$

and an array decision $d_{\text {a }}$ given by

$$
d_{\mathrm{a}}=\operatorname{sgn}\left(\sum_{m=1}^{M} v_{\mathrm{a}_{m}}\right) \text {. }
$$

6) Intra-Domain Fusion of Quantitative and Qualitative Features on Decision Level: The intra-domain combination of quantitative and qualitative features on decision-level is based on the Boosting approach [27]. The purpose of this method is to combine $K$ possibly unreliable classification hypotheses $H_{k}$ to an improved hypothesis $\hat{H}$ by a weighted sum

$$
\hat{H}=\sum_{k=1}^{K} \alpha_{k} H_{k}, \quad \alpha_{k} \geq 0 .
$$

The hard decision $d_{\mathrm{h}}$ is created from the signs of the differences $v_{\delta_{i}}$ in (14) and the qualitative feature vector $\boldsymbol{v}_{\mathrm{ql}}$ by

$$
d_{\mathrm{h}}=\operatorname{sgn}\left(\sum_{i} \operatorname{sgn}\left(v_{\delta_{i}}\right)+\sum_{i} v_{\mathrm{ql}_{i}}\right) \text {. }
$$

The weighted decision $d_{\mathrm{w}}$ is created with

$$
d_{\mathrm{w}}=\operatorname{sgn}\left(\sum_{i} v_{\delta_{i}}+\sum_{i} v_{\mathrm{ql}_{i}}\right)
$$

where the absolute value of $v_{\delta_{i}}$ is used as implicit weighting.

\section{B. Space-Domain Multipath Detection}

Using an antenna array, also multiple independent information-theoretical model order estimators can be used to calculate the number of received signals from $R_{Y Y}$ in (12) in the space domain. The intra-domain fusion of these numerical results is conducted by an evidence approach. As model order estimators the Akaike information criterion $(A I C)$ [28], the minimum description length $(M D L)$ [29], the modified $M D L(\bmod M D L)$, and the minimum probability of error criterion (MPEC) [30] are considered. Each of these methods is based on the eigenvalue decomposition of the spatial covariance matrix $R_{Y Y}$ and results in a likelihoodbased signal-number estimate. All methods assume spatially uncorrelated signals. The estimates of the different model order estimators are combined by an evidence approach, known as the Dempster-Shafer Theory of Evidence [24]. We use simplified equations from [31].

1) Evidence Based Intra-Domain Fusion of Different Model Order Estimates: For an antenna array with $M$ elements, the $M$ possible signal-number hypotheses are $h_{j}=j-1, \forall j \in$ $[1, M]$. As we assume that $L<M-1$ the hypotheses include the true number of signals. Further, to each estimation method $m_{i}$, a certain basic belief probability $\psi_{i} \in(0,1)$ is assigned, as a measure of trust into its results. The $\psi_{i}$ have to be derived from the false alarm rates in multipath-free conditions, however, for simplicity, we assume all $\psi_{i}$ to be known. Using the signal-number estimates $D_{i}$ of all methods, the combined evidences $f_{j}$ in favor of each hypothesis $h_{j}$ is

$$
f_{j}=1-\prod_{i}\left(1-e_{i, j}\right),
$$

where the evidence $e_{i, j}$ of each method $m_{i}$ for each hypothesis $h_{j}$ is given by

$$
e_{i, j}=\left\{\begin{array}{l}
\psi_{i} \text { if } D_{i}=h_{j} \\
0 \text { otherwise }
\end{array}\right.
$$

Since there are no explicit evidence against hypotheses, the belief masses $B_{j}$ for the hypotheses $h_{j}$ are calculated as

$$
B_{j}=\frac{f_{j}}{1-f_{j}} \cdot \frac{1}{1+\sum_{k=1}^{M} \frac{f_{k}}{1-f_{k}}} .
$$

The combined space-domain signal-number estimate $\hat{D}$ is the hypothesis with maximum belief mass, i.e

$$
\hat{D}=\arg \max _{j} B_{j}
$$


The corresponding detection result $d_{\mathrm{s}}$ is given by

$$
d_{\mathrm{s}}=\left\{\begin{array}{l}
+1 \text { if } \hat{D}>1 \\
-1 \text { if } \hat{D}=1 \\
0 \text { otherwise. }
\end{array}\right.
$$

\section{Inter-Domain Fusion of Temporal and Spatial Decision}

The decisions $d_{\mathrm{q}}(15), d_{\mathrm{a}}(19), d_{\mathrm{h}}(21), d_{\mathrm{w}}(22)$, from the time-domain and the decision $d_{\mathrm{s}}(27)$ from the space-domain can be combined by logical operations. First, the inter-domain fusion with the $A N D$-combination $d_{\mathrm{AND}}$ is given by

$$
d_{\mathrm{AND}}=\left\{\begin{array}{l}
1 \text { if } d_{\text {time }}=1 \wedge d_{\text {space }}=1, \\
-1 \text { otherwise }
\end{array}\right.
$$

and results in a detection only if multipath is detected in all domains. Second, the inter-domain $O R$-combination $d_{\mathrm{OR}}$ is

$$
d_{\mathrm{OR}}=\left\{\begin{array}{l}
1 \text { if } d_{\text {time }}=1 \vee d_{\text {space }}=1, \\
-1 \text { otherwise }
\end{array},\right.
$$

which results in a detection if multipath is detected in at least one domain. For simplicity, we now omit the error-handling in case of $d_{\text {time }}=0$ or $d_{\text {space }}=0$. Depending on the detector's optimization target, the fusion type can be chosen.

\section{Simulation Results}

The performance of the previously described multipath detection methods is evaluated with simulated GNSS signals. As performance metrics the detection rate $r_{\mathrm{det}}$, which corresponds to the probability of correctly classifying a MP reception situation and the false alarm rate $r_{\mathrm{fa}}$ which corresponds to the probability of falsely classifying a non-MP situation as multipath, are evaluated [32], [33], [34]. They are defined as

$$
r_{\mathrm{det}}=\frac{N_{\mathrm{TP}}}{N_{\mathrm{TP}}+N_{\mathrm{FN}}},
$$

and

$$
r_{\mathrm{fa}}=\frac{N_{\mathrm{FP}}}{N_{\mathrm{TN}}+N_{\mathrm{FP}}},
$$

where $N_{\text {TP }}$ is the number of true positives (MP situations correctly classified as MP situations), $N_{\mathrm{FP}}$ is the number of false positives (non-MP situations falsely classified as MP situations), $N_{\mathrm{TN}}$ is the number of true negatives (non-MP situations correctly classified as non-MP situations) and $N_{\mathrm{FN}}$ is the number of false negatives (MP situations falsely classified as non-MP situations).

The GNSS signals are generated with a software signal generator and a hardware signal generator. For all scenarios we assume an uniform rectangular array (URA) antenna based GNSS receiver with $M=4$ elements and $Q=5$ correlators. The correlators are at positions $\hat{\tau}_{Q}=\left[-0.75 T_{\mathrm{c}},-0.25 T_{\mathrm{c}}, 0 T_{\mathrm{c}}, 0.25 T_{\mathrm{c}}, 0.75 T_{\mathrm{c}}\right]^{\mathrm{T}}$ with respect to the estimated LOS delay. The single-sided bandwidth is $B=8.184 \mathrm{MHz}$, i.e., the sampling rate is $f_{s}=16.368 \mathrm{MHz}$. For the estimation of $\boldsymbol{R}_{\boldsymbol{Y} \boldsymbol{Y}}$, post-correlation samples $\boldsymbol{Y}[k]$ of $K_{p}=50$ consequent observation periods are collected.

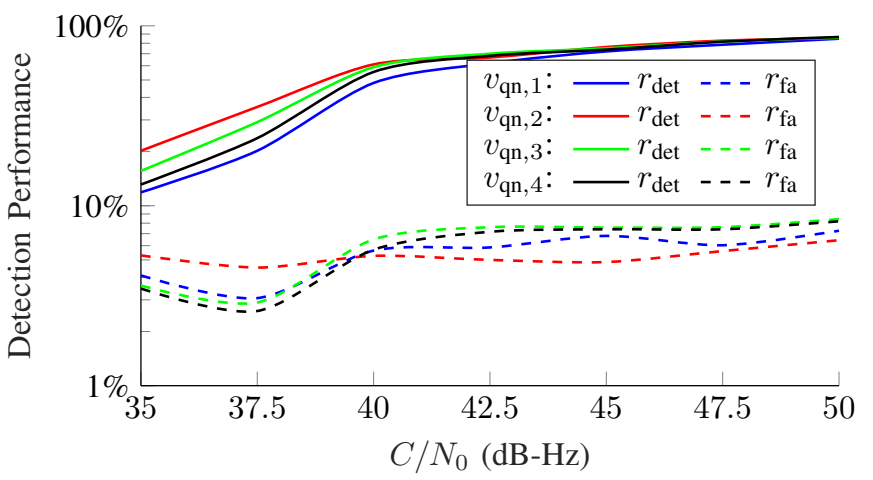

Fig. 1. Comparison of detection and false alarm rates of the quantitative features $v_{\mathrm{qn}_{1}}, v_{\mathrm{qn}_{2}}, v_{\mathrm{qn}_{3}}, v_{\mathrm{qn}_{4}}$.

In the time-domain, the results are averaged over the same observation window. Its size was determined empirically as a tradeoff between spatial resolution and temporal alterations.

\section{A. Software-based simulations}

For the software simulated signals, $180 \mathrm{~s}$ long sequences containing one GNSS signal are generated. Periodically, an attenuated, phase-shifted and delayed replica signal is superposed. The signal parameters for both direct and indirect signals are shown in Table I.

TABLE I

SIGNAL PARAMETERS FOR SOFTWARE-BASED SIMULATIONS

\begin{tabular}{|l|l|l|l|l|l|}
\hline Signal & Atten. & $\boldsymbol{\tau}$ & $\boldsymbol{\varphi}$ & $\boldsymbol{\theta}$ & $\boldsymbol{\phi}$ \\
\hline Direct & $0 \mathrm{~dB}$ & $0 T_{c}$ & $0^{\circ}$ & $42^{\circ}$ & $260^{\circ}$ \\
Indirect & $6 \mathrm{~dB}$ & $0.2 T_{c}$ & $180^{\circ}$ & $72^{\circ}$ & $150^{\circ}$ \\
\hline
\end{tabular}

1) Performance Comparison of Multipath Detection with Time Domain Features: Fig. 1 shows the detection and false alarm rates of the quantitative features $v_{\mathrm{qn}_{1}}, v_{\mathrm{qn}_{2}}, v_{\mathrm{qn}_{3}}, v_{\mathrm{qn}_{4}}$ listed in Table IV in the appendix over $\mathrm{C} / \mathrm{N}_{0}$. For all features the detection performance increases with $\mathrm{C} / \mathrm{N}_{0}$, while the false alarm rate is always below $10 \%$.

Fig. 2 shows the detection and false alarm rates for the combined decisions $d_{\mathrm{q}}(15), d_{\mathrm{h}}(21)$, and $d_{\mathrm{w}}(22)$. Compared to the single-feature case, the intra-domain combination lead to slightly increased detection rates for $C / N_{0}$ above 40 $\mathrm{dB}-\mathrm{Hz}$, while having similar, false alarm probabilities.

2) Performance Comparison of Multipath Detection with Model Order Estimation: Fig. 3 shows the detection and false alarm rates of the model order estimators $A I C, M D L$, modMDL, MPEC. All methods achieve approximately the same detection rate. The false alarm rate of MPEC is by a factor 10 lower than the false alarm rate of the time domain methods. The false alarm rates of $A I C, M D L, \bmod M D L$ are below $1 \%$ and therefore not shown in the plot. In comparison 


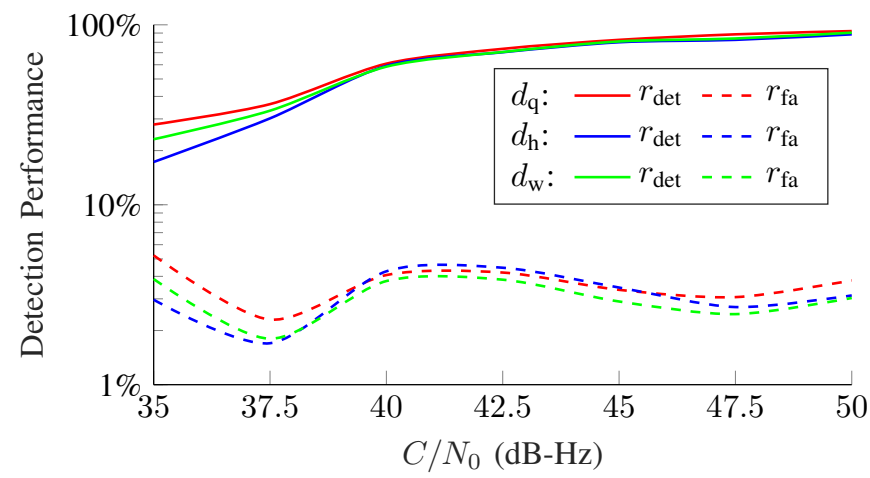

Fig. 2. Comparison of detection and false alarm rates of the quantitative, hard and weighted decision $d_{\mathrm{q}}(15), d_{\mathrm{h}}(21)$, and $d_{\mathrm{w}}(22)$.

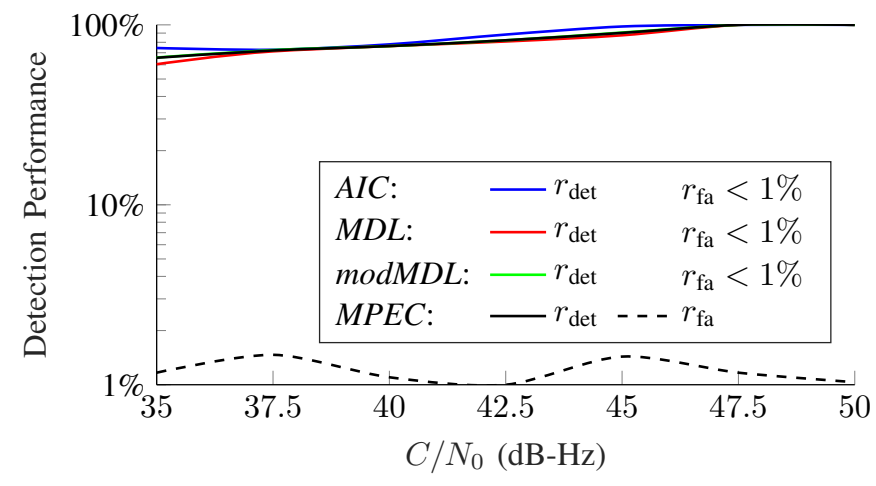

Fig. 3. Comparison of detection and false alarm rates of the model order estimators $A I C, M D L, \bmod M D L, M P E C$.

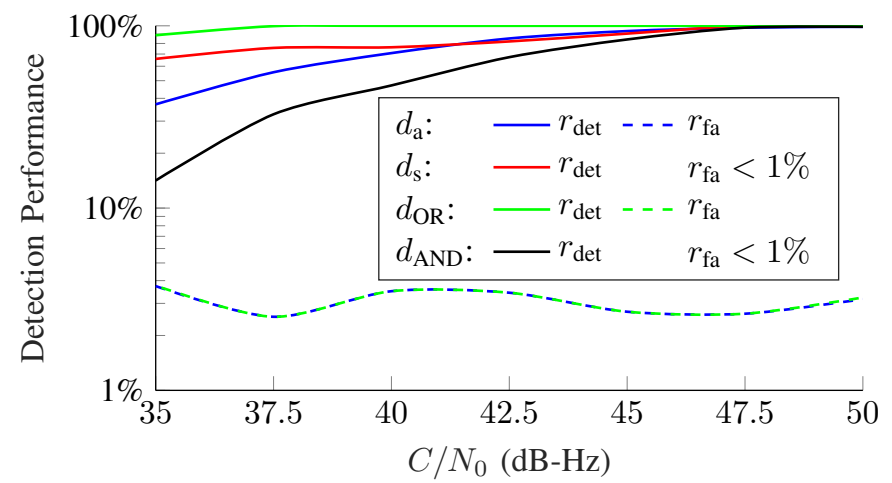

Fig. 4. Comparison of detection and false alarm rates of the array based fusion of $d_{\mathrm{a}}(19), d_{\mathrm{s}}(27), d_{\mathrm{OR}}(29)$, and $d_{\mathrm{AND}}(28)$

to the time-domain multipath detection methods all model order estimators achieve a much smaller false alarm rate.

Fig. 4 shows the performance of different combined methods. The intra-domain combination of model order estimates $d_{\mathrm{s}}$ achieves a false alarm rate lower than $1 \%$ and a higher detection rate than the single model order estimates. The time-domain quantitative decision, averaged over the array, $d_{\mathrm{a}}$, achieves a significant increase in detection

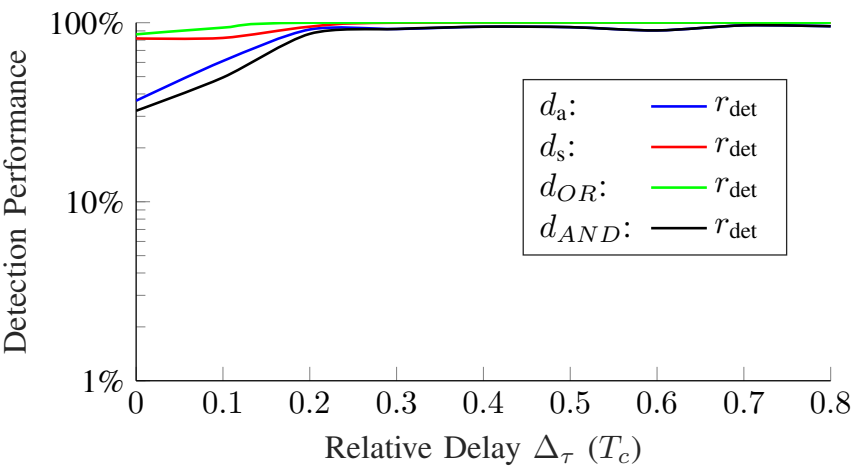

Fig. 5. Comparison of detection and false-alarm rates of $d_{\mathrm{a}}(19), d_{\mathrm{s}}(27)$, $d_{\mathrm{OR}}(29)$, and $d_{\mathrm{AND}}(28)$

rate of nearly $50 \%$ points at $\mathrm{C} / \mathrm{N}_{0}=35 \mathrm{~dB}-\mathrm{Hz}$, while the false alarm rate remains at $2-4 \%$ in to the single-antenna time-domain methods. The logical inter-domain combinations perform as expected: for the $A N D$-combined decision $d_{\mathrm{AND}}$ no false alarms are detected in the simulated scenario, while the detection rate is reduced. The $O R$-combined decision $d_{\mathrm{OR}}$ achieves a high detection rate at the cost of a higher false alarm probability. The latter is determined by the highest false alarm rate of the combined decisions, i.e., $d_{\mathrm{a}}$.

To analyze the impact of different signal properties, additional scenarios are analyzed, where one at a time the indirect signals' attenuation, the relative time delay between LOS and multipath and the multipath angle of arrival are varied. The LOS signal $C / N_{0}$ is $45 \mathrm{~dB}-\mathrm{Hz}$ while the other parameters for the direct signal are the ones listed in Table I. The false alarm rates are not dependent on the multipath parameters and are therefore not considered in the following. They are in theory the same as in Fig. 4.

Fig. 5 shows the detection rates of $d_{\mathrm{a}}, d_{\mathrm{s}}, d_{\mathrm{OR}}$, and $d_{\text {AND }}$ over the relative time delay between LOS and multipath signal. Especially for the time-domain quantitative decision, averaged over the array, $d_{\mathrm{a}}$, and the $A N D$ combined decision $d_{\mathrm{AND}}$ the detection rate decreases strongly for short-range multipath of 0-0.2 chips distance to the LOS signal.

Fig. 6 shows the detection rates of $d_{\mathrm{a}}, d_{\mathrm{s}}, d_{\mathrm{OR}}$, and $d_{\mathrm{AND}}$ over the signal-to-multipath ratio (SMR) signal. For all methods the detection rate equally drops with decreasing reflection power, but nevertheless, in both variation scenarios, the $O R$-combined inter-domain decision $d_{\mathrm{OR}}$ is able to guarantee detection rates in the area of $85-100 \%$.

Fig. 7 shows the detection rate of the time-domain quantitative decision, averaged over the array, $d_{\mathrm{a}}$. As expected for $d_{\mathrm{a}}$ the detection rate is flat over all angles of arrival, as the time domain features do not dependent on the direction. Fig. 8 shows the detection rate of the combined model order estimate decision $d_{\mathrm{s}}$. The detection rate shows a minimum 


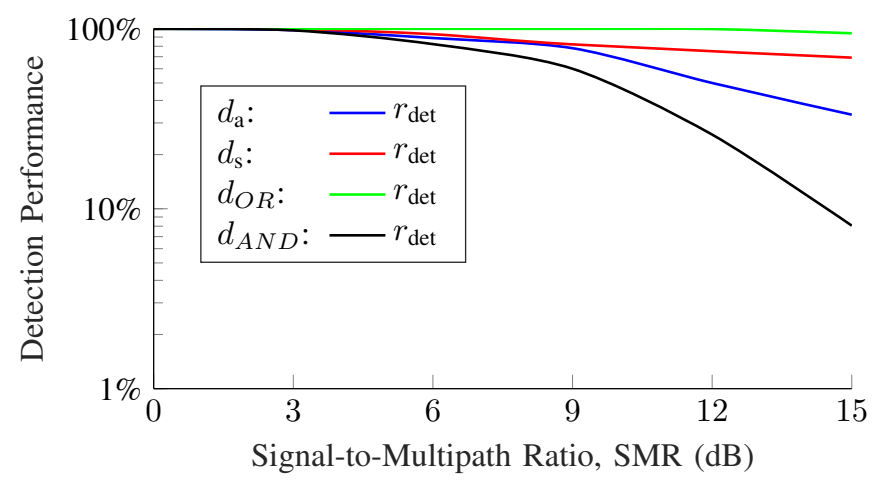

Fig. 6. Comparison of detection and false alarm rates of $d_{\mathrm{a}}(19), d_{\mathrm{s}}(27)$, $d_{\mathrm{OR}}(29)$, and $d_{\mathrm{AND}}(28)$

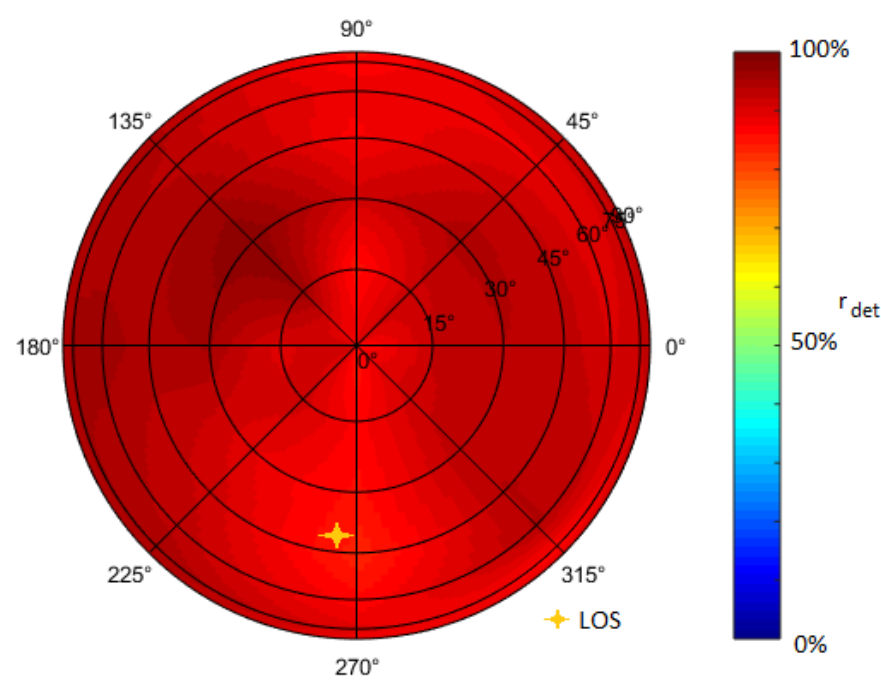

Fig. 7. Detection rate of $d_{\mathrm{a}}$ (19) over angle of arrival of multipath signal

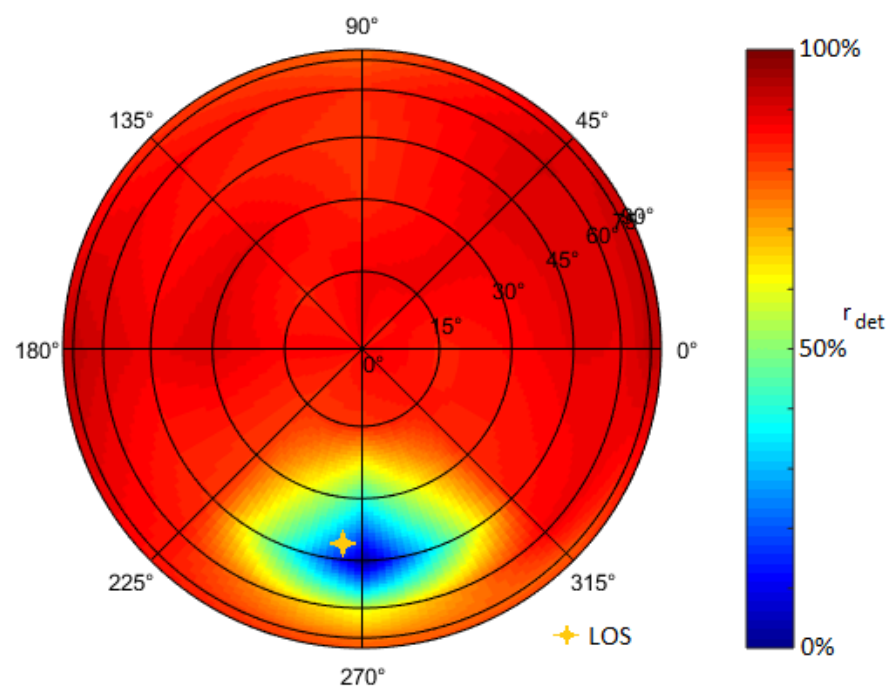

Fig. 8. Detection rate of $d_{\mathrm{s}}$ (27) over angle of arrival of multipath signal

around the coordinates of the LOS signal. In this case the signals are spatially correlated, which violates one of the

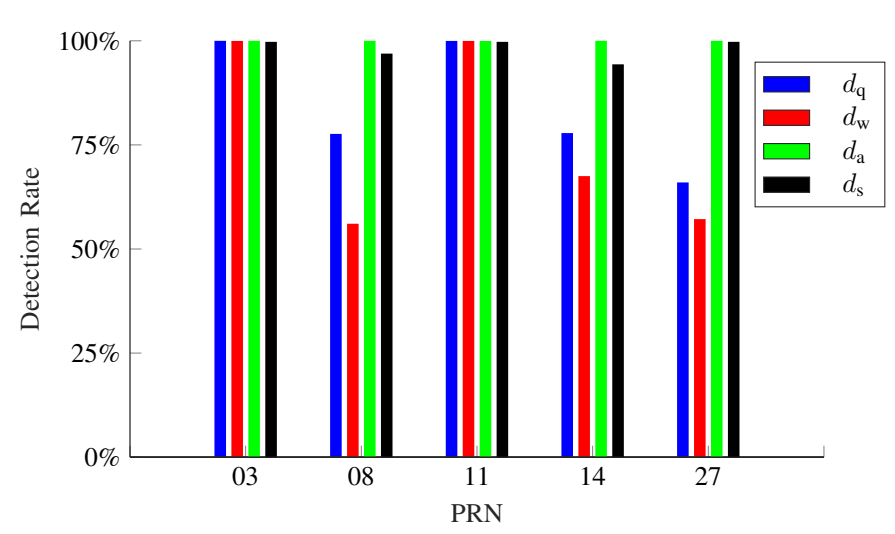

Fig. 9. Detection rates for hardware simulated signals for $d_{\mathrm{q}}, d_{\mathrm{w}}, d_{\mathrm{a}}$ and $d_{\mathrm{s}}$

assumptions of the space based model order estimators. Considering $d_{\mathrm{OR}}$ would clearly show, that the decisions of different domains can be combined to increase the overall multipath detection rate.

\section{B. Hardware-based simulations}

For the hardware-based simulation, the radio frequency (RF) signal received of a $2 \times 2$ URA is simulated for a constellation of five satellites with the Spirent GSS9000 GNSS simulator with 4 RF outputs. The signal is mixed to an intermediate frequency of $75 \mathrm{MHz}$ with an analogue front-end [35] and recorded with the National Instruments datagrabber described in [36]. For each satellite, a simple multipath model is applied, which generates one signal echo from the same elevation, but with a mirrored azimuth angle. We recorded $420 \mathrm{~s}$ long signals, start with multipath-free conditions. After $113 \mathrm{~s}$, the multipath echos are switched on, while after additional 180 seconds, the previously used direct signals are switched off. The last part of this scenario corresponds to the situation, where the LOS signals are shielded and only indirect signals are observed. The reference signal power is $48 \mathrm{~dB}-\mathrm{Hz}$ for the direct signals. The parameters for the indirect signals are shown in Table II. Fig. 9 shows the

TABLE II

ECHO SIGNAL PARAMETERS FOR HARDWARE-BASED SIMULATIONS

\begin{tabular}{|l|l|l|l|l|}
\hline Satellite & Atten. & $\boldsymbol{\tau}$ & $\boldsymbol{\theta}$ & $\boldsymbol{\phi}$ \\
\hline PRN03 & $6 \mathrm{~dB}$ & $0.341 T_{c}$ & $61^{\circ}$ & $47^{\circ}$ \\
PRN08 & $7 \mathrm{~dB}$ & $0.512 T_{c}$ & $65^{\circ}$ & $279^{\circ}$ \\
PRN11 & $8 \mathrm{~dB}$ & $0.682 T_{c}$ & $78^{\circ}$ & $319^{\circ}$ \\
PRN14 & $5 \mathrm{~dB}$ & $0.273 T_{c}$ & $58^{\circ}$ & $163^{\circ}$ \\
PRN27 & $5 \mathrm{~dB}$ & $0.205 T_{c}$ & $50^{\circ}$ & $240^{\circ}$ \\
\hline
\end{tabular}

detection rate for the time-domain quantitative decision $d_{\mathrm{q}}$, the time-domain weighted decision $d_{\mathrm{w}}$, the time-domain quantitative decision, averaged over the array, $d_{\mathrm{a}}$ and the combined model order estimates decision $d_{\mathrm{s}}$. The detection rate is highly satellite-dependent. In the case of PRN03 and PRN11 the indirect echos are sufficiently delayed relative to the LOS signal. Therefore,Compared to the single-antenna 


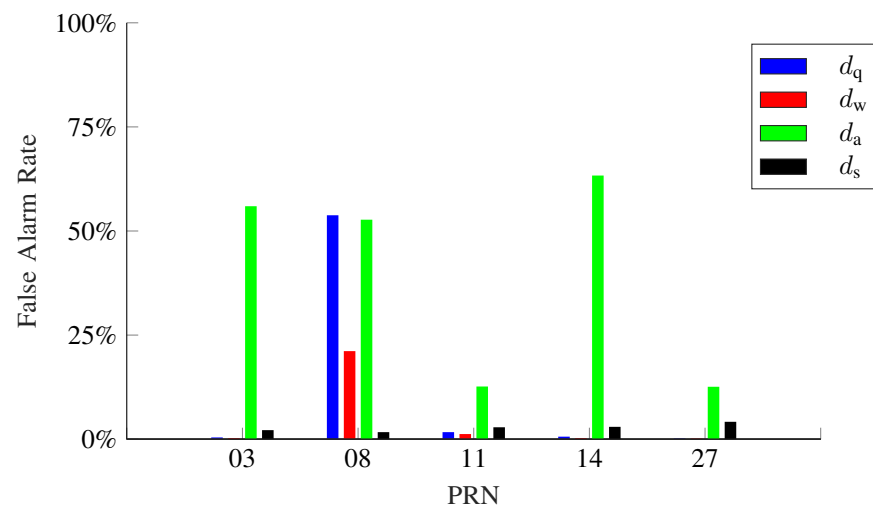

Fig. 10. False alarm rates for hardware simulated signal for $d_{\mathrm{q}}, d_{\mathrm{w}}, d_{\mathrm{a}}$ and $d_{\mathrm{s}}$

time-domain methods, they are detected by both time-domain and space-domain methods. The multipath signals from PRN14 and PRN27 have a lower relative delay to the LOS signal. Thus they are more difficult to separate by the time domain methods $d_{\mathrm{q}}$ and $d_{\mathrm{w}}$.

Fig. 10 shows the false alarm rates for $d_{\mathrm{q}}, d_{\mathrm{w}}, d_{\mathrm{a}}$ and $d_{\mathrm{s}}$. In comparison to the results from the software-generated simulations, $d_{\mathrm{a}}$ exhibits a high false alarm rate of partly more than $50 \%$. Also the combined model order estimates from the space domain $d_{\mathrm{s}}$, show higher false alarm rates, in comparison to the software-based simulations. The outlying false alarm rates for PRN08 of the time domain methods $d_{\mathrm{q}}$ and $d_{\mathrm{w}}$ may be explained by the shape of the PRN's cross-correlation function [10].

\section{Conclusion}

In this paper, we presented a multipath detection method which uses an intra-domain combination of multiple detection criteria, followed by an inter-domain fusion approach. In the time-domain, we used a classification approach with selected CCF features, which we combined either at featureor at decision-level and additionally averaged over multiple elements of the array antenna. In the space-domain, we used different signal-number estimators and combined them by an evidence approach. Finally, we performed logical inter-domain fusion to receive combined results.

Regarding the time-domain, simulation results show that the intra-domain fusion results in an increased detection rate and a reduced false-alarm rate, compared to single-feature decisions. The presence of multiple decision criteria relaxes the need of knowledge of single-feature performance in different receiver conditions, thus creating additional invariance and robustness. As expected, the time-domain methods exhibits weaknesses in temporally correlated conditions, which can be resolved by inter-domain fusion, i.e., by averaging over the elements of an array antenna.
The overall detection performance of the space-domain methods is higher than the one of the time-domain methods, considering both detection and false alarm rates. The latter can be further reduced by the chosen evidence-based fusion approach without significant loss of detection probability. For spatially correlated signals the space-domain methods fail to detect multipath, which can be solved by inter-domain fusion.

The $O R$-based inter-domain fusion maximizes detection rates by equalizing the hard conditions for either working domain, at the cost of increased false alarm rates. In comparison, the $A N D$-based fusion is able to reduce the false alarm rate to insignificant values, at the cost of reduced detection rate.

Still remaining challenges are the determination of feature distributions for the time-domain and basic belief probabilities for the space-domain. The approach presented in our work required careful receiver calibration in LOS conditions, which can be difficult in realistic environments and in case of changed receiver settings. In the future, the empirical calibration could be replaced by analytic models of the receiver behavior for increased robustness. Additionally, an improvement could be made by investigating different feature combination types, for example by introducing soft-decisions or continuous weighting schemes.

\section{APPENDIX A \\ Qualitative Features}

The qualitative features evaluate the CCF for extreme deviations. The features which are used in this work are summarized in Tab. III, where $I_{q}$ denotes the inphase, i.e. real value of the $q$-th correlator output of the considered antenna, i.e the real part of the $q$-th column of $\boldsymbol{Y}[k]$ in the row of the considered antenna. The dependence on $k$ is omitted for the sake of readability. Further, $\dot{I}_{q}$ denotes the derivative of the CCF at the $q$-th correlator which is calculated by $\dot{I}_{q}=\frac{I_{q+1}-I_{q}}{\hat{\tau}_{q+1}-\hat{\tau}_{q}}$.

\section{APPENDIX B QuANTITATIVE FEATURES}

The quantitative features evaluate the relative asymmetry or the deviation from the theoretical shape of the CCF. The features which are used in this work are summarized in Tab. IV. Note, that in the ideal case all features are 0.

\section{ACKNOWLEDGMENT}

The research leading to these results has been carried out under the framework of the project $R \& D$ for the maritime safety and security and corresponding real time services. The project started in January 2013 and is led by the Program Coordination Defence and Security Research within the German Aerospace Center (DLR). 
TABLE III

LIST OF QUALITATIVE MULTIPATH FEATURES IN THE TIME DOMAIN

\begin{tabular}{|l|l|}
\hline 1 & Prompt correlator is larger than early correlators \\
\hline \hline 2 & $v_{\mathrm{ql}_{1}}=\left\{\begin{array}{l}1 \text { if } \exists i \in[1, P) \mid I_{i} \geq I_{P}, \\
0 \text { else. }\end{array}\right.$ \\
\hline Early side increases monotonically
\end{tabular}

\section{REFERENCES}

[1] E. Kaplan and C. Hegarty, Understanding GPS: principles and applications. Artech house, 2005.

[2] M. Irsigler, G. W. Hein, and B. Eissfeller, "Multipath performance analysis for future GNSS signals," in Proceedings of the 2004 National Technical Meeting of The Institute of Navigation, Jan. 2004.

[3] M. Z. H. Bhuiyan and E. S. Lohan, "Multipath mitigation techniques for satellite-based positioning applications," in Global Navigation Satellite Systems: Signal, Theory and Applications. InTech, 2012.

[4] Y. Zhang and C. Bartone, "Multipath mitigation in the frequency domain," in Proc. PLANS 2004. Position Location and Navigation Symposium, pp. 486-495.

[5] O. M. Mubarak and A. G. Dempster, "Analysis of early late phase in single-and dual-frequency GPS receivers for multipath detection," GPS solutions, vol. 14, no. 4, pp. 381-388, 2010.

[6] F. Schweppe, "Sensor-array data processing for multiple-signal sources," IEEE Transactions on Information Theory, vol. 14, no. 2, pp. 294-305, Mar. 1968.

[7] G. Seco-Granados, J. A. Fernandez-Rubio, and C. Fernandez-Prades, "ML estimator and hybrid beamformer for multipath and interference mitigation in GNSS receivers," IEEE Transactions on Signal Processing, vol. 53, no. 3, pp. 1194-1208, Feb. 2005.

[8] M. Brenneman, J. Morton, F. van Grass, and C. Yang, "Mitigation of GPS multipath using polarization and spatial diversities," in Proceedings of the 20th International Technical Meeting of the Satellite Division of The Institute of Navigation, 2007, pp. 1221 - 1229.

[9] F. Fohlmeister, A. Iliopoulos, M. Sgammini, F. Antreich, and J. Nossek, "Dual polarization beamforming algorithm for multipath mitigation in gnss," Signal Processing, vol. 138, pp. 86-97, 2017.

[10] R. E. Phelts, "Multicorrelator techniqueas for robust mitigation of threats to GPS signal quality," Ph.D. dissertation, Stanford University, 2001.

[11] M. Irsigler and G. W. Hein, "Development of a real time multipath monitor based on multi-correlator observations," in Proceedings of the
TABLE IV

LIST OF QUANTITATIVE MULTIPATH FEATURES IN THE TIME DOMAIN

\begin{tabular}{|c|c|}
\hline 1 & Derivative of early side isequal to derivative of late side \\
\hline & $v_{\mathrm{qn}_{1}}=\frac{I_{P}-I_{1}}{\hat{\tau}_{P}-\hat{\tau}_{1}}-\frac{I_{Q}-I_{P}}{\hat{\tau}_{Q}-\hat{\tau}_{P}}$. \\
\hline \multirow[t]{2}{*}{2} & Derivative of early side is flat \\
\hline & $v_{\mathrm{qn}_{2}}=\dot{I}_{1}-\dot{I}_{P-1}$ \\
\hline \multirow[t]{2}{*}{3} & Derivatives at both sides of the prompt correlatorare equal \\
\hline & $v_{\mathrm{qn}_{3}}=\left|\dot{I}_{P-1}\right|-\left|\dot{I}_{P}\right|$ \\
\hline \multirow[t]{2}{*}{4} & Sum of derivatives on both sides of the prompt correlatorare equal \\
\hline & $v_{\mathrm{qn}_{4}}=\frac{1}{P-1} \sum_{i=1}^{P-1}\left|\dot{I}_{i}\right|-\frac{1}{P-1} \sum_{i=P}^{Q-1}\left|\dot{I}_{i}\right|$ \\
\hline \multirow[t]{2}{*}{5} & Derivative of late side is flat \\
\hline & $v_{\mathrm{qn}_{5}}=\dot{s}_{p}-\dot{s}_{Q-1}$ \\
\hline \multirow[t]{2}{*}{6} & Standard deviation of derivatives is 0 \\
\hline & $v_{\mathrm{qn}_{6}}=\sqrt{\frac{1}{Q-1} \sum_{i=1}^{Q-1}|| \dot{I}_{i}|-\mu|^{2}}$ \\
\hline \multirow[t]{2}{*}{7} & Mean of derivatives is 0 \\
\hline & $v_{\mathrm{qn}_{7}}=\frac{1}{Q-1} \sum_{i=1}^{Q-1}\left|\dot{I}_{i}\right|-1$ \\
\hline \multirow[t]{2}{*}{8} & Derivatives should be 1 \\
\hline & $v_{\mathrm{qn}_{8}}=\sum_{i=1}^{Q-1}\left(\left|\dot{I}_{i}\right|-1\right)^{2}$ \\
\hline \multirow[t]{2}{*}{9} & Area below CCF matches ideal case \\
\hline & $\begin{aligned} v_{\mathrm{qn}_{9}}= & \sum_{i=1}^{Q-1}\left[\left(\hat{\tau}_{i+1}-\hat{\tau}_{i}\right)\left(I_{i}+I_{i+1}\right)\right]- \\
& \sum_{i=1}^{Q-1}\left[\left(\hat{\tau}_{i+1}-\hat{\tau}_{i}\right)\left(2-\hat{\tau}_{i}-\hat{\tau}_{i+1}\right)\right]\end{aligned}$ \\
\hline
\end{tabular}

18th International Technical Meeting of the Satellite Division of The Institute of Navigation (ION GNSS 2005), Long Beach, CA, Sep. 2005.

[12] M. Z. H. Bhuiyan, E. S. Lohan, and M. Renfors, "Code tracking algorithms for mitigating multipath effects in fading channels for satellitebased positioning," EURASIP Journal on Advances in Signal Processing, vol. 2008 , no. 1 , p. 863629, 2007.

[13] _ "A slope-based multipath estimation technique for mitigating short-delay multipath in GNSS receivers," in Proceedings of the IEEE International Symposium on Circuits and Systems, Paris, France, May 2010, pp. 3573-3576.

[14] A. J. van Dierendonck, P. Fenton, and T. Ford, "Theory and performance of narrow correlator spacing in a GPS receiver," Navigation, vol. 39, no. 3, pp. 265-283, 1992.

[15] M. S. McGraw, G. A .and Braasch, "GNSS multipath mitigation using gated and high resolution correlator concepts," in Institute of Navigation, National Technical Meeting'Vision 2010: Present and Future', San Diego, CA, Jan. 1999, pp. 333-342.

[16] R. D. J. van Nee, "The multipath estimating delay lock loop," in Proc. IEEE Second Int. Symp. Spread Spectrum Techniques and Applications, Yokohama, Japan, Nov. 1992, pp. 39-42.

[17] M. Wax and T. Kailath, "Detection of signals by information theoretic criteria," IEEE Transactions on Acoustics, Speech, and Signal Processing, vol. 33, no. 2, pp. 387-392, Apr. 1985. 
[18] R. Schmidt, "Multiple emitter location and signal parameter estimation," IEEE Transactions on Antennas and Propagation, vol. 34, no. 3, pp. 276-280, 1986.

[19] R. Roy and T. Kailath, "ESPRIT-estimation of signal parameters via rotational invariance techniques," IEEE Transactions on Acoustics, Speech, and Signal Processing, vol. 37, no. 7, pp. 984-995, Jul. 1989.

[20] P. Stoica and K. C. Sharman, "Novel eigenanalysis method for direction estimation," IEE Proceedings F - Radar and Signal Processing, vol. 137, no. 1, pp. 19-26, Feb. 1990.

[21] S. U. Pillai and B. H. Kwon, "Forward/backward spatial smoothing techniques for coherent signal identification," IEEE Transactions on Acoustics, Speech, and Signal Processing, vol. 37, no. 1, pp. 8-15, Jan. 1989.

[22] B. Townsend and P. Fenton, "A practical approach to the reduction of pseudorange multipath errors in a L1 GPS receiver," in Proceedings of the 7th International Technical Meeting of the Satellite Division of the Institute of Navigation, Salt Lake City, UT, USA, Jan. 1994.

[23] J. Ohm, Multimedia Content Analysis, ser. Signals and Communication Technology. Springer, 2016.

[24] G. Shafer, A mathematical theory of evidence. Princeton university press, 1976, vol. 42.

[25] E. L. Lehmann and J. P. Romano, Testing statistical hypotheses. Springer Science \& Business Media, 2006.

[26] D. L. Hall and J. Llinas, "An introduction to multisensor data fusion," Proceedings of the IEEE, vol. 85, no. 1, pp. 6-23, 1997.

[27] R. E. Schapire and Y. Singer, "Improved boosting algorithms using confidence-rated predictions," in Proceedings of the eleventh annual conference on Computational learning theory. ACM, 1998, pp. 80-91.
[28] H. Akaike, "A new look at the statistical model identification," IEEE Transactions on Automatic Control, vol. 19, no. 6, pp. 716-723, Dec. 1974.

[29] J. Rissanen, "Modeling by shortest data description," Automatica, vol. 14 , no. 5, pp. 465-471, Sep. 1978.

[30] D. B. Williams, "Comparison of aic and mdl to the minimum probability of error criterion," in Conference Proceedings of the IEEE Sixth SP Workshop on Statistical Signal and Array Processing, Victoria, Canada, Aug. 1992, pp. 114-117.

[31] J. A. Barnett, "Computational methods for a mathematical theory of evidence." in IJCAI, vol. 81, 1981, pp. 868-875.

[32] C. E. Metz, "Basic principles of ROC analysis," in Seminars in nuclear medicine, vol. 8, no. 4. Elsevier, 1978, pp. 283-298.

[33] D. G. Altman and J. M. Bland, "Statistics notes: Diagnostic tests 2: predictive values," BMJ: British Medical Journal, vol. 309, no. 6947, p. 102, Jul. 1994.

[34] T. Fawcett, "An introduction to ROC analysis," Pattern recognition letters, vol. 27, no. 8, pp. 861-874, Jun. 2006.

[35] M. V. T. Heckler, M. Cuntz, A. Konovaltsev, L. A. Greda, A. Dreher, and M. Meurer, "Development of Robust Safety-of-Life Navigation Receivers," IEEE Transactions on Microwave Theory and Techniques, vol. 59, no. 4, pp. 998-1005, \#apr\# 2011.

[36] A. Iliopoulos, F. Fohlmeister, F. Antreich, M. Appel, E. P. Marcos, M. Sgammini, and M. Cuntz, "Testing of a multi-antenna gnss receiver in dlr's maritime jamming testbed," in Deutscher Luftund Raumfahrtkongress 2016, September 2016. [Online]. Available: http://elib.dlr.de/113090/ 